\title{
Synthesis and Characterization of New Dental Composite for Dentistry Based on Unsaturated Monomers and Nano-fillers by Photopolymerization
}

\author{
Mahdi Saleh Mahdi ${ }^{*}$, Mohammed Ali Mutar ${ }^{a}$ \\ ${ }^{a}$ Chemical Engineering Department -Faculty of Engineering - University of Al-Qadisiyah-Iraq
}

\section{ART ICLE INFO}

Article history:

Received 03 October 2019

Received in revised form 06 January 2020

Accepted 23 January 2020

\section{Keywords:}

Dental

composite

Nano fillers

\begin{abstract}
A B S T R A C T
In this study, dental nano-composite specimens were prepared by dispersion of various amounts of nanosized fillers ( $\mathrm{HA}, \mathrm{ZrO}_{2}$, and $\mathrm{SiO}_{2}$ ) in a monomer system containing 60\% Bis-GMA and 40\% TEGDMA. 2,2 propyl bis-phenyl glycidyldimethacrylate (Bis-GMA) with unsaturated monomers were prepared. Camphor Quinone (CQ) of $1 \mathrm{wt} \%$, 2-DiMethyl Amino Ethyl Methacrylate (DMAEMA) of $1 \mathrm{wt} \%$ have been applied in photo-initiation system for the purpose of initiating matrix resins' co-polymerization. Wear resistance, flexural strength, hardness and compressive strength were measured. The results indicated an increase in the mechanical properties in the samples containing nano-size filler particles. It is interesting to note that, this improvement was observed at much lower nano-size filler content. Physicochemical properties, such as Solubility (SL), Water Sorption (WS) as well as the Volumetric Shrinkage (VS) have been examined. FT-IR as well as SEM have been utilized for implementing the characterization. SEM has been applied for showing particle size distribution as well as the particle agglomeration that is related to the treated nano-fillers in nano-composites. FT-IT is initially applied for identifying qualitative compositions regarding the nano-composites' compositions. The Thermal stability of all dental nano-ocomposites was also studied using TGA and DSC techniques.
\end{abstract}

\section{Introduction}

Using dental composite for the purposes of filling material is becoming widespread because of the need for more esthetic filling materials, also due to the laws banning amalgam in certain nations. Amalgam, considered as filling materials, was utilized for over one-hundred and fifty years; also, in many places of the world it is still applied. It can be defined as alloy of mercury as well as other metal including copper, tin, in addition to silver.
Over the years, there was a debate regarding if amalgam can be considered toxic or not toxic, yet, this debate is still not settled [1]. A major drawback of applying dental composite material is that they have micro-mechanically retentions to the surfaces of the tooth, and that makes under-cuts needless for the composite filling, yet not in the case of applying amalgam. On the basis of combinations of various particles as well as their filler size, the

* Corresponding author. Tel.: +964(0) 7705545252. 
dental composites could be categorized. Also, their viscosity is used to classify them.

There are 2 major phases related to composites: inorganic and organic. There are various types related to monomers included majorly in nonpolymerized organic matrix. The major monomers applied in composite include Bis-GMA, TEGDMA as well as UDMA. The composite has high viscosity due to the heaviness and stiffness of Bis-GMA. TEGDMA can be defined as molecule that is small and flexible, also its molecular weight is low, and that make its viscosity less in comparison to Bis-GMA, also it is more possible to be subjected to greater polymerization shrinkage.

The viscosity of UDMA is low in comparison to Bis-GMA, also it could be utilized alone, however it is frequently combined with certain other monomers in the composites that are used currently, for the purpose of improving different performances [2]. To add inorganic glass filler particles is another approach for reducing shrinkage. Maximal filler loadings could be reached via utilizing various sized shaped as well as sized filler particles. The composite's consistency is impacted via the shape, size and amount of the fillers. The small particle as nano-fillers, provide high gloss in the case when polishing the composites which will lead to optimum esthetics $[3,4]$. Recently, the size of the filler particles has been small and the majority of composites used today consist of small amount of nano-particles.

The majority of dental composites are considered to be hybrids, and they can be categorized to nano-hybrid or micro-hybrid composites according to their filler load as well as their filler size. By definition, there are filler particles from at least 2 filler size range are included in each one of the composites. The size range of the particles is in $(10 \mathrm{~nm}-10 \mu \mathrm{m})$. The range of the filler particles that are relate to micro filled composites is between $10 \mathrm{~nm}$ and $50 \mu \mathrm{m}$. Nano-composites include cluster or particles of the size range $(1 \mathrm{~nm}-1.4 \mathrm{~nm})$ [2,4]. Nano-materials could be defined according to European Commission, no less than fifty percent of particles should be in size range (1nm - 100nm) [5]. The process of curing is initiated via the curing light. In the case of applying light, monomer polymerize and create rigid structures referred to as polymer networks.

Throughout the polymerization process, double bonding will be converted to single bonding between carbon molecules. The distance between molecules will be shortened because of such conversion, which will lead to polymerization shrinkage. Following polymerization process, the composites will be converted to solid structures. Polymerization shrinkage might result in more sensitivity, secondary caries, stress, cracks, dis-coloring, and gaps in the restored tooth [4,6,7]. Stress which is created via the VS at tooth-restoration interface will create marginal gaps, that unfavorably impact the resin composite restoration's longevity. There are many aspects could impact the polymerization shrinkage such as the prepolymerized particles, filler particles type, filler load, monomer systems, and so on. [8]. The resin composite's VS is detected via the resin matrix compositions as well as the filler content [9]. Also, Kleverlaan et al. showed that the resins in resin composites will be decreased with the increase in filler load which will result in reduction in shrinkage [10].

With regard to the presented study, the aim is to check the 5 monomers that can enhance the mechanical characteristics as well as the polymer network structures, in the case when co-polymerized. For the purpose of achieving such task, various compositions, majorly applied in dentistry field, that consist of TEGDMA as well as Bis-GMA in addition to unsaturated monomer (Methyl methacrylate, (2-Hydroxyethyl)_ methacrylar, Triethylene glycol dimethacrylate, 2,2 propyl bisphenyl glycidyldimethacrylate), have been altered through the co-polymerizing process, Bis-GMA with the un-saturated monomers. Furthermore, SL, flexural modulus, WS, hardness, VS, wear resistance, and SEM morphology have been examined.

FT-IR and SEM have been applied for implementing the characterization. SEM has been utilized for showing particle agglomerations as well as particle size distribution that are related to the treated nano-fillers in nano-composite. FT-IR has been initially applied for identifying qualitative compositions regarding the compositions of the nano-composites. Thermal stability that is related to all the dental nanocomposites has been examined through the use of DSC and TGA methods.

\section{Experimental work}

\subsection{Materials and system}

Methyl methacrylic (MMA) (MERCK), (2-Hydroxyethyl)_ methacrylar (2-HEMA)( MERCK), 2,2 propyl bisphenyl glycoldimethacrylate (Bis-GMA)(USA), Triethylene glycol dimethacrylate (TEGDMA)( Aldrich), Zinc Oxide (ZnO)( GCC), Zirconium Oxide Nanoparticles ( $\mathrm{ZrO} 2)($ Skyspring Nanomaterial), Silicon Oxid Nanoparticles (SiO2)( Skyspring Nanomaterial), Nano Hydroxy Apatite (HA)( Skyspring Nanomaterial), 2-(Diethyl amino)ethyl acrylate(ALDRICH), Camphorquinone(ALDRICH).

\subsection{Apparatuses}

Digital sensitive balance, Sarorius, B1210s, Germany, Mixter, Scanning Electron Microscopy (SEM)(JEOL ,Japan), FTIR TENSOR 27, Fourier transform infrared spectroscope, BRUKER, Germany., (Thermogravimetry Analysis (TGA) were performed on a polymer laboratories co England, Model pL-TG at Iran polymer and petrochemical institute, using a heating rate of $10^{\circ} \mathrm{C} / \mathrm{min}$ in argon atmosphere within the temperature range of (25$800^{\circ} \mathrm{C}$ ). Differential thermal analysis is measured using apparatus (DSC) type (DSC 131 Evo, SETARAM ,France) in the college of education for pure science Ibn - AL Haitham central service laboratory/University of Baghdad. Properties are measured using a tensile testing machine from ( LARYEE Co)/ China under a load cell of $20 \mathrm{KN}$ and a cross-head speed of $200 \mathrm{~mm} / \mathrm{min}$ at room temperature. The sample dimensions were accordance to ASTM D-412.

\subsection{Fabrication of Experimental Dental Nano-composites (A1-A3)}

The experimental dental nano-composites have 2 series, which have been subjected to a process of fabrication through mixing fillers as well as the monomer matrix. Monomer matrix (Bis-GMA, 2-HEMA, MMA, and TEGDMA) has been subjected to mixing process in the mass ratio (40/20/20 and 20) for one hour. After that, a process of adding the zinc oxide $(0.5 \mathrm{gm})$ as antimicrobial agent has been implemented, also a process of adding a nano-filler $(0.7 \mathrm{gm})$ as a colorant has been also implemented for improving the mechanical characteristics. After that, a process of adding DMAEMA (0.5 wt $\%)$ as accelerator and camphor quinone (0.5 wt $\%)$ as initiator has implemented and continuous for twenty minutes. Then, the paste has been inserted to the test moulds and the light cured with the use of a light curing unit (EliparFreelight2LED, $3 \mathrm{M} \mathrm{ESPE)} \mathrm{at} 1500 \mathrm{~mW} / \mathrm{cm}^{2}$ intensity. Light has been illuminated on the two surfaces, bottom and top, via clear matrix strips for forty seconds. Since it is majorly applied via clinicians, curing time of forty seconds has been applied for curing the experimental nano-composites. Tip distance that is related to light curing unit has been kept at 1-2 $\mathrm{mm}$ from the surface of the surface. The concentration of materials is explained in Table 1. 
Table 1: Monomers and photo initiators used in this study

\begin{tabular}{|c|c|c|c|c|c|c|c|}
\hline \multirow[t]{2}{*}{ Sample No. } & \multirow[t]{2}{*}{ BisGMA(\%) } & \multirow[t]{2}{*}{ 2-HEMA(\%) } & \multirow[t]{2}{*}{ MMA(\%) } & \multirow[t]{2}{*}{ TEGDMA(\%) } & \multirow{2}{*}{$\begin{array}{l}\text { Nano fillers } \\
\text { (0.7 gm) }\end{array}$} & \multicolumn{2}{|l|}{ Initiator } \\
\hline & & & & & & Camphorq-uinone & DMAEMA \\
\hline A1 & 40 & 20 & 20 & 20 & $\mathrm{SiO}_{2}$ Nano & $0.5 \mathrm{wt} \%$ & $0.5 \mathrm{wt} \%$ \\
\hline A2 & 40 & 20 & 20 & 20 & $\mathrm{ZrO} 2$ Nano & $0.5 \mathrm{wt} \%$ & $0.5 \mathrm{wt} \%$ \\
\hline A3 & 40 & 20 & 20 & 20 & Hydroxy aptiate Nano & $0.5 \mathrm{wt} \%$ & 0.5 wt $\%$ \\
\hline
\end{tabular}

\subsection{The Process of Curing}

The monomers have been mixed with: 0.4wt\% Camphor Quinone (CQ, Sigma-Aldrich) the photo-sensitizer and $1 \mathrm{wt} \% \mathrm{~N}, \mathrm{n}$-DiMethylAminoEthylMethacrylate (DMAEMA, Sigma-Aldrich) the reducing factor and with vigorous stirring, poured to moulds Petri dishes (with a diameter of $120 \mathrm{~mm}$ and thickness of $4 \mathrm{~mm}$ ). Those samples have been covered by PET film for the sake of reducing the impacts of the inhibition of oxygen and after that, irradiated, at a room temperature, for half an hour. Photo-polymerization has been started by a mercury vapor lamp of high pressure (FAMED1, model L6/58, Lodz, Poland, power 375W $[11,12])$, which emits Ultra-Violet/VIS light, where camphorquinone is absorbed in a range between $420 \mathrm{~nm}$ and $500 \mathrm{~nm}$ [13].

\subsection{Measurement of Volumetric Shrinkage (VS)}

The VS related to dental resins has been examined via the variations in density pre and post the process of photo polymerization, also, the Archimedes principle is used to determine the density. Measurement is carried out with the use of commercial density determination kits related to the analytical balance Mettler ToledoX on basis of ISO17304:2013 (E).

\subsection{Measurement of Water Sorption (WS) and Solubility (SL)}

Water Sorption (WS) and Solubility (SL) have been acquired on the basis of ISO4049 [14].

\subsection{Scanning Electron Microscopy (SEM)}

Researches on the Morphology have been carried out on the cured materials' fractured surfaces with Hitachi TM3000 SEM, Tokyo, Japan. Sample surfaces, prior to observations, have been sputter coated in gold.

\subsection{Physico-mechanical properties of the test samples}

\subsubsection{Flexural characteristics}

The flexural strength $(\sigma)$ and the flexural modulus (E) are specified according to the ISO178 .

\subsubsection{Compression set}

Compression set was measured according to ISO 1653 at ambient temperature.

\section{Results and Discussions}

\subsection{Prepared Polymers Synthesis and Characterization}

\subsubsection{Synthesis and FTIR spectrum}

The preparation of DNC (A) has been done via co-polymerization process that is related to the 2-HEMA, MMA, Bis-GMA, with cross-linker (TEGDMA), initiator (KPS), the reaction was continued through a process of refluxing for two hours and at a temperature of 26 Celsius under $\mathrm{N}_{2}$ gas for the purpose of removing dissolved oxygen, just as can be seen in reaction scheme (1):

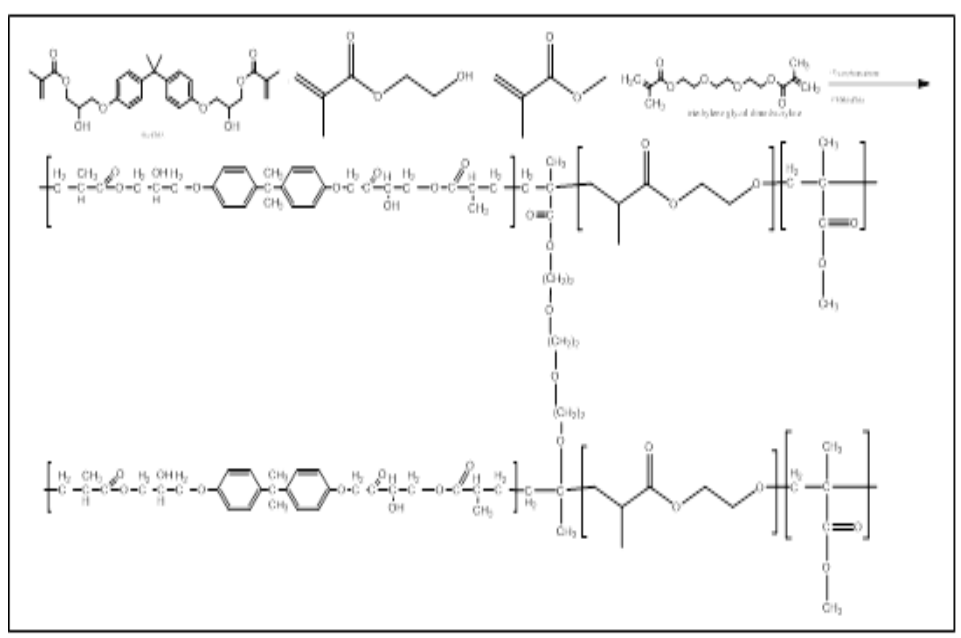

Scheme 1: Synthesis of polymer (A)
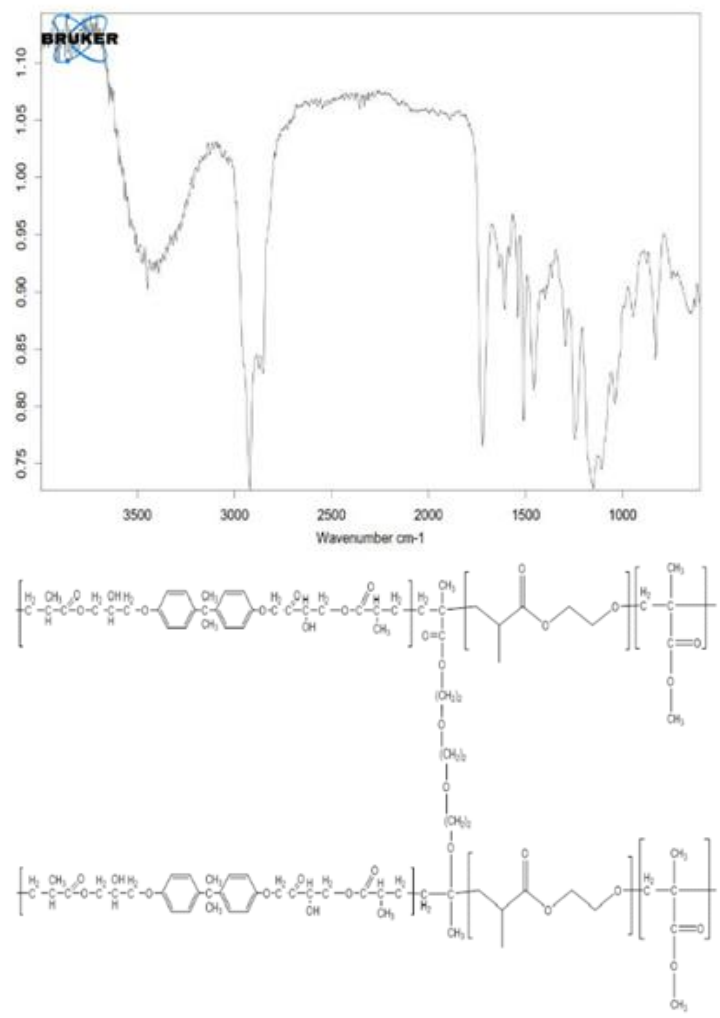

Figure 1. FTIR spectra for (A) 


\section{FTIR analysis}

There are many bands related to the infrared spectrum, majorly the wide range in range of (3300 - 3450) $\mathrm{cm}-1$, that indicate overlaps the current absorption $(\mathrm{OH})$. Appearance of the beams in range of $(2850 \mathrm{~cm}-1$ $2990 \mathrm{~cm}-1)$ to vibratory vibration that is related to aliphatic $\mathrm{CH}$ bonds in polymer's structure. Characteristic beams at $1725 \mathrm{~cm}-1$ indicate $(\mathrm{C}=\mathrm{O})$ of ester's group, aromatic $(\mathrm{C}=\mathrm{C})$ are indicated at range of $(1475-1600 \mathrm{~cm}$ 1). Characteristic bands in range of $(1390 \mathrm{~cm}-1-1400 \mathrm{~cm}-1)$ are the result of $(\mathrm{C}-\mathrm{H})$ bonds for the $(\mathrm{CH} 3)$ group of polymers. The absorption bands in range of (1000 - 1100cm- 1$),(\mathrm{C}-\mathrm{O})$ as can be seen in Fig. 1:

\subsubsection{Water Sorption and Solubility (WS)}

Water Sorption (WS) and Solubility (SL) have been acquired on the basis of ISO4049 [14]. Steel split mold has been utilized for making the specimens with diameter of ( 15 millimeter) and ( 1 millimeter) thick $(n=5)$. WS has been decreased reduced through using extra hydrophobic monomer, preventing the hygroscopic expansion. Water sorption might be accounted for the adverse effects such as discoloration and subsequent swelling of restorative materials.

With regard to the present study, there has been an increase in the water sorption with the reduction in conversions of the tested formulation, because of their molecule's hydrophobic character, as well as the elevated conversion, Bis-GMA presented with the lowest water sorption [15]. With regard to the N,N MBAA, elevated conversions do not translate to elevated cross-linking density, as mentioned earlier, thus second to last lowest water sorption has been fairly unpredicted [16], also it should be associated with its low hydrophilicity in comparison to Bis-GMA. Very comparable water sorption values have been shown by hydrophobic monomers and BisGMA, despite differences in the conversion, possibly due to the fact that these are considered to be the major hydrophilic molecules assessed in the present study.

In addition to being dependent on hydrophilic character and amount of leachable in products, the results of solubility are on the basis of amount of water absorbed in network. Furthermore, the pendant double bonds which are contributing to greater free volume to network [17] and favor WS, could not be contributing to the leachable species (oligomers and monomers), due to the fact that they're tied to network. This might explain the reason behind solubility values being related to Bis-GMA have not been greatest among homopolymers, regardless of its considerably more elevated WS. N,N MBAA, at the same time, because of tendency to cyclization, indicated the highest results of SL, despite low water sorption and high conversion, probably due to the fact that the oligomers of lowmolecular-weight have been existent and ready for leaching. The low SL results from the Bis-GMA have been clarified through the hydrophobic character as well as the high conversion (with regard to this condition, possibly also with greater cross-linking) of molecule. Also, it must be indicated that the results of SL could be undervalued for more hydrophilic monomer, due to the fact that the water yield stronger hydrogen bond interaction with the hydroxyl and (in Bis-GMA), as well as weak bond with the ethylene glycol unit (which are presented in great concentrations in $\mathrm{N}$, $\mathrm{N}$ MBAA ), the could be hindering the water elimination throughout the 2nd period of storage in desiccators [16]. It appears that the distinct characteristics which are related to tested dimethacrylate homopolymers are the cause of their unique behavior with regard to the flexural properties, polymerization kinetics, WS and SL. These properties explain using the copolymers for the purpose of obtaining high DC and mechanical characteristics, in addition to optimum resistance to water degradation.
It can be indicated that, before water immersion of Bis-GMA-based resin would be similar after water immersion, which can be an indication of its more effective water resistance. WSL reveal the amount of the unreacted monomers which are leached out of the polymeric networks. The major cause of tissue inflammation as well as cytotoxicity is the monomers' release [18]. It has been indicated that Degree of conversion (DC) and WSL are related, and low WSL could be the result of high DC [19]. This must be based on un-reacted monomers' leachability in polymeric networks. Intermolecular hydrogen bond that is created via - $\mathrm{NH}$ is weaker than the hydrogen bond created via - $\mathrm{OH}$, due to the $-\mathrm{OH}$ group's high cohesive energy density [19]. Thus, unreacted monomers can undergo a process of absorption to surrounding networks more firmly in Bis-GMA based polymers in addition to being to leach out of polymers, which might lead to low WSL regarding the Bis-GMA based polymers. Bis-GMA monomers result in more hydrophobic molecule; thus, the materials will have less susceptibility to liquid's sorption as can be seen in Fig. 2 .

Since the polydimethacrylates can be considered as cross-linked dental polymers, and because of the polymer chains have cross-links between them, this will typically lead to considerable reduction in polymer's solvent permeability since the reduce the capability related to polymer chain for swelling as well as reducing hole free volume [20]. Nevertheless, WS could be defined as needed impact as the composites' hygroscopic expansion was indicated to the close marginal leakage gap because of the shrinkage [21]. At the same time, a significant standard for the dental composites is the sufficient resistance to degradation via water and other solvents. It has been indicated that the dental composites are leaching only (0.02-0.05 percent) of their total mass to the aqueous solution through the first thirty days, this is the result of inorganic nano-fillers.

\subsubsection{Volumetric Shrinkage}

The Volumetric Shrinkage (VS) values which are related to the specimens of the composites have been assessed on the basis of Archimedes principle. Uncured sample has been left to rest for one minute for the purpose of eliminating the impact of the slumping on measurements, then it has been exposed to 40s light curing, and the curing unit tip was placed at a distance of (1-2 millimeter) from specimen. The assessment is implemented through the use of commercial density determination kit of analytical balance Mettler Toledo X on the basis of ISO17304:2013 (E).

One of the main disadvantages, particularly in the dental composites is that their polymerization shrinkage could result in secondary caries and marginal gaps in restored teeth. Shrinkage is caused by the composites' matrix-phase polymerization, also it is a main disadvantage in the dental resin-monomer. The polymerization of resin is accompanied by the volumetric shrinkage. As the polymerization proceeds, van deer Waals distances have been modified to covalent bond distance which results in volumetric shrinkage. The shrinkage degree is represented through the number of the created covalent bonds that is defined as the polymerization reaction's extent. Another reason for the volumetric shrinkage is that the molecular distances between polymer chains become smaller than the molecular distance between monomers [22].

Since the stresses generated from polymerization shrinkage cause defects of deboning at the tooth-restorative interface [23,24], one of the main issues in the dental composites is to eliminate or decrease the polymerization shrinkage. In the case when the monomer in the proximity react to create covalent bonds, there will be a reduction in the distance between atoms groups, also the free volume will be decreased, that will lead to VS magnitude experienced through composites has been decided through its filler volume fraction as well as the conversion's degree and composition 
of resin matrix. As shown in Fig. 3, the composite with the Bis-GMA presents much smaller volumetric shrinkage. The shrinkage values which were indicated for Bis-GMA (forty percent) and N,N MBAA (twenty percent) have been considerably more elevated than the shrinkage values related to the standard composites, that are in the range of $2-3$ percent $[25,26]$. Such difference is the result of the fact that in the hybrid composite, about sixty percent of volume has been occupied through filler particle. Even though that the inorganic content related to micro filled composites is approximately forty percent, their shrinkage values are considered to be comparable to hybrids, because of the existence of pre-polymerized composite particle, occasionally indicated as "organic fillers", that will be rendering them in a way comparable to the hybrid composite with regard to actual volume fraction regarding the polymerizing resin. The shrinkage will also be affected via the diluents' concentrations in resin matrix.

A new study indicated that high ratios of N,N MBAA /Bis-GMA in the experimental composite will lead to high contraction stress value because of the elevated VS, due to the improved conversions [27]. Due to the fact that they usually have low molecular weights in comparison to host monomers, the density of the polymerizable carbon double bonds will be increased via the "diluents" monomers that will result in more shrinkage. Also, the reaction environment's mobility will increase because of the $\mathrm{Tg}$ as well as low viscosity of diluent, which will allow more effective conversions [28]

Previous studies showed that increasing light intensity resulted in more polymerization shrinkage for some composites, but not all materials [29,30]. In our study, the effects of each curing mode on two composite resins were compared where no significant difference was observed. Each composite showed similar behavior in the face of modulation of the light intensity during photoactivation. Therefore, it must be put into perspective that this result is valid only for two resin composites in this study. Consequently, with regard to this study limitations, it might be indicated that the increase in the shrinkage of composites is related to the total energy of curing lights. Further research in this area is necessary in order to reveal the polymerization shrinkage of other composite resins.

\subsubsection{Scanning Electron Microscopy}

Monodispersed and spherical nano-particles were successfully synthesized. SEM is used to evaluate both the morphology of nanoparticles and fillers composite surface following the fracture toughness test (SEM, LEO (Zeiss) 1540XB). Samples were prepared for SEM by placing a small amount of the sample powder on SEM tubes followed by coating with osmium in order to prevent charging of the samples and to reduce the nano-tube damage from the electron beam during imaging. Surface treatment has been of high importance to develop and efficient dental composites. In the case when the filler's size is not more than thirty nm, agglomerations as well as aggregation of the nano-particles will occur. Thus, interfacial adhesions between the filler and the polymer matrix will be improved via the agglomeration filler dispersion. The improvements in the mechanical and surface characteristics might be the result of these enhancements. With regard to the presented study, zirconium oxide as well as nano-silica have been applied as filler, that has been mixed with various concentrations chosen of the monomer resin in addition to the additives for the dental restorative applications. Using zirconium oxide fillers and nanosilica have been anticipated to have benefits and enhance the characteristics related to composite fabricated. Klapdhor and Moszner 14 have indicated that the mono-dispersed nano-filler in polymer matrix result in nanocomposite material of optimum mechanical characteristics, excellent process ability as well as high transparency.
DNC A1. In this DNC (Bis-GMA, 2- HEMA, MMA) monomers at different concentration were used and crosslink (TEGDAA) at 20\% was used after that $0.7 \mathrm{gm} \mathrm{SiO} 2$ and $0.5 \mathrm{gm} \mathrm{ZnO} 2$ were added. Fig. 4(a) shows that spherical shapes with different size range of $29.4-90.6 \mathrm{~nm}$ of nano silica composite with monomer were obtained. SEM micrograph of each material is shown in Fig. 4 (a and b) at two different magnifications.
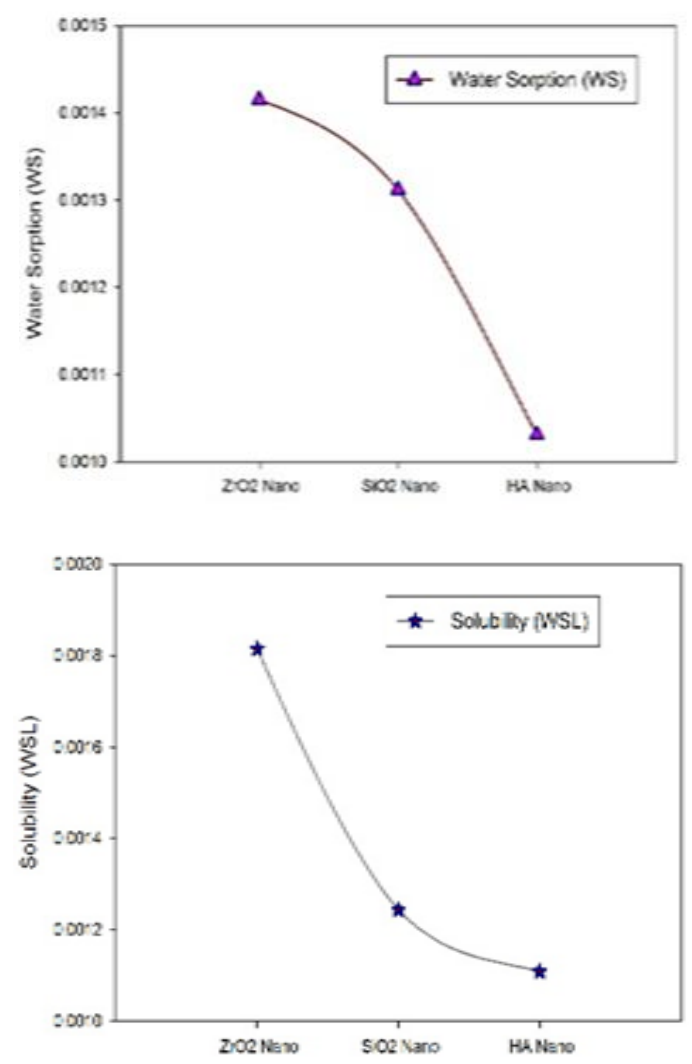

Figure 2. Water Sorption (WS) and water Solubility (SL)

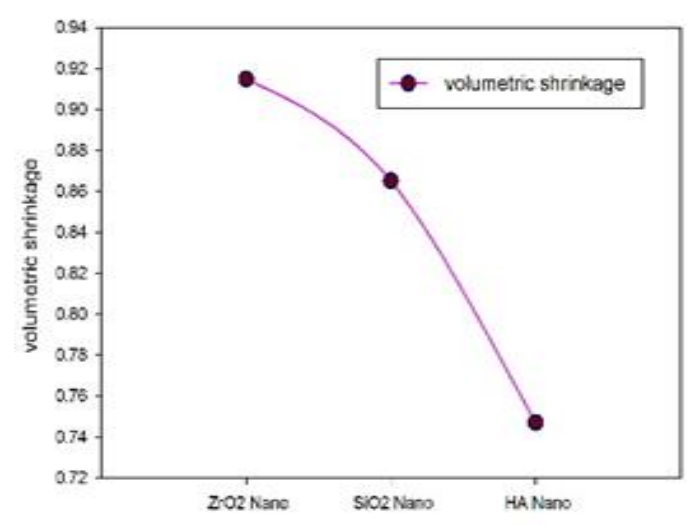

Figure 3. Volumetric Shrinkage 


\section{DNC A1}
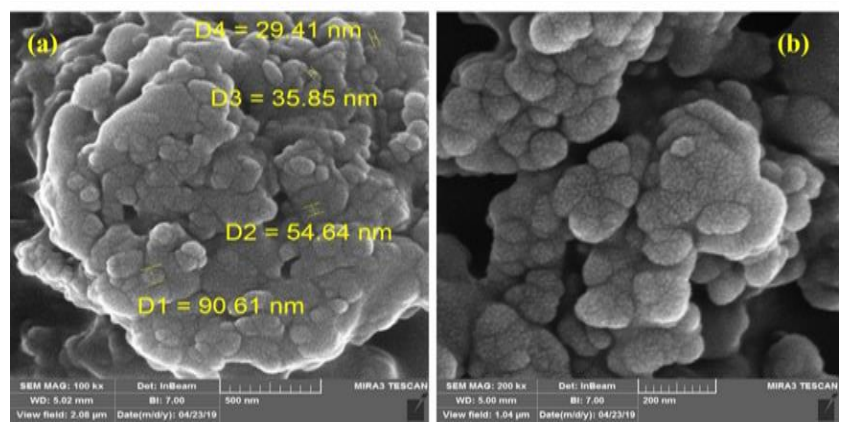

Figure 4 (a and b): SEM image of Nanosilica composite

In DNC A2, the same monomers are used as in DNC A1 but it differs only in nano-filler that was used of $0.7 \mathrm{gm} \mathrm{ZrO} 2$ is added. Fig. 5 (a) shows that spherical shapes with different size range of $75.7-106.9 \mathrm{~nm}$ of zirconium oxide nanoparticles composite with monomer were obtained. SEM micrograph of each material is shown in Fig. 5 ( $a$ and b) at two different magnifications.

\section{DNCA2}
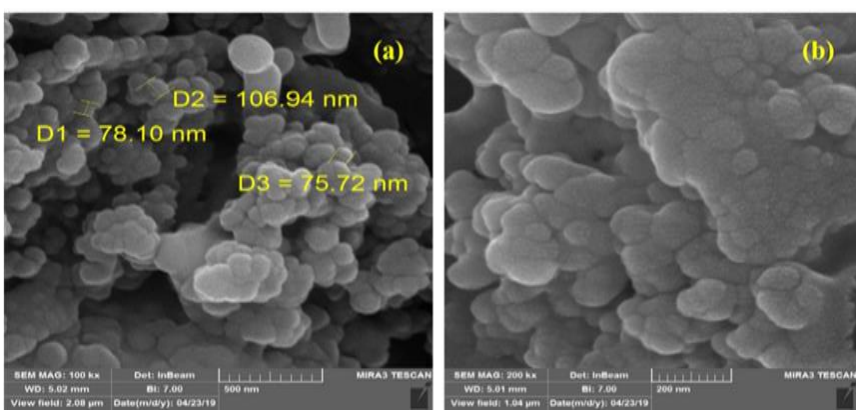

Figure 5 (a and b): SEM image of zirconium oxide nano-particles composite

\subsubsection{Thermal gravimetric analysis (TGA) study}

Thermogravimetric Analysis determines the change in mass as temperature's functions. It can be mainly applied to specify degradation temperature, material's absorbed content, the level of inorganic as well as the organic parts in the materials as well as residues of analysis solvent. It does use sensitive electronic balance through which a sample will be suspended in furnace, which is controlled through temperature programmer. Thermal characteristics of 4 samples of such polymers have been examined by TGA in Argon atmosphere at heating rate $\left(10^{\circ} \mathrm{C}\right.$ per minute) [31-33]. With regard to this test, many values have been recognized including Ti, Top, Tf, T50\%, Residue at 600 Celsius, and char yields at 400 Celsius as can be seen in Table 2.

Temperatures of fifty percent weight loss of (DNC A1- DNC A3) of polymers have been between $(384-409){ }^{\circ} \mathrm{C}$, The char results of (DNC A1) are $79 \%$,(DNC A2) are $78 \%$, (DNC A3) are $77 \%$ at $500{ }^{\circ} \mathrm{C}$ in argon atmosphere, indicating that they could satisfy the requirements of temperature resistance that may be utilized in a variety of applications. Weight residue of (DNC A1) are 81.5\%, (DNC A2) are 80\%, (DNC A3) are $79 \%$, at $600{ }^{\circ} \mathrm{C}$.
Table 2: Thermal Stability Characteristics Curves, Thermal Gravimetric Analysis (TGA) of polymers represents the temperature of decomposition.

\begin{tabular}{lccccll}
\hline DNC & $\mathbf{T}_{\mathbf{i}}$ & $\mathbf{T}_{\text {op1 }}$ & $\mathbf{T}_{\mathbf{f}}$ & $\mathbf{T}_{\mathbf{5 0 \%}}$ & $\begin{array}{l}\text { Residue } \\
\text { at }^{\mathbf{0}} \mathbf{C 6 0 0}\end{array}$ & $\begin{array}{l}\text { Char \% At } \\
\mathbf{5 0 0}{ }^{\circ} \mathbf{C}\end{array}$ \\
\hline A1 & 318 & 351 & 456 & 384 & 81.5 & 79 \\
\hline $\mathbf{A 2}$ & 278 & 330 & 440 & 385 & 80 & 78 \\
A3 & 280 & 350 & 455 & 409 & 79 & 77 \\
\hline
\end{tabular}

\section{DNC Dental Nano Composite}

$\mathrm{T}_{\mathrm{op} 1}$ represents optimal temperature of decomposition.

$\mathrm{T}_{\mathrm{i}}$ represents the temperature of the initial decomposition.

$\mathrm{T}_{50 \%}$ represents the temperature of $50 \%$ weight loss, which has been obtained from the TGA.

$\mathrm{T}_{\mathrm{f}}$ represents the final temperature of decomposition. The final degree of dissociation temperature

Char\% at $400{ }^{\circ} \mathrm{C}$ represents the residual weight percentage at $500^{\circ} \mathrm{C}$ in argon by TGA.

\subsubsection{Differential Scanning Calorimeter Analysis (DSC) Study}

Differential Scanning Calorimetry (DSC), is a technique of thermal analysis that investigates how material's heat capacity $(\mathrm{Cp})$ is transformed by temperature. A known mass sample is heated or cooled and the variations in its heat capacity are observed as alterations in the heat flow. This allows to reveal transitions such as melts glass transitions (Tg), and the melting point $(\mathrm{Tm})$ the crystallization degree (Tc) (20). The results of (DNC A1) polymer showed in Fig.6. The value of the glass transition (Tg) of the mixture was $110^{\circ}$ an increase in the flow of temperature and then increase the rate of absorption of the sample to the temperature until the melting point $(\mathrm{Tm})$ at $\left(584^{\circ} \mathrm{C}\right)$ if completely melted and then the rate of absorption of the sample to heat and by the crystallization rate (Tc) of the mixture was set at $\left(150.3^{\circ} \mathrm{C}\right)$. The results of (DNC A2) showed in Fig.6. The value of the glass transition $(\mathrm{Tg})$ of the mixture was $111^{\circ} \mathrm{C}$ indicating an increase in the flow of temperature and then increase the rate of absorption of the sample to the temperature until the melting point (Tm) at $\left(580^{\circ} \mathrm{C}\right)$ if completely melted and then the rate of absorption of the sample to heat and the crystallization rate (Tc) of the mixture was set at $\left(145.4^{\circ}\right.$ C). The results of (DNC A3) polymer showed in Fig. 6. The value of the glass transition $(\mathrm{Tg})$ of the mixture was $106^{\circ} \mathrm{C}$ indicating an increase in the flow of temperature and then increase the rate of absorption of the sample to the temperature until the melting point $(\mathrm{Tm})$ at $\left(584^{\circ} \mathrm{C}\right)$ if completely melted and then the rate of absorption of the sample to heat and the crystallization rate $(\mathrm{Tc})$ of the mixture was set at $\left(11138^{\circ} \mathrm{C}\right)$ [34].

Table 3: Degree of Glass Transition, Melting Point and the Degree of Crystallization in the Differential Thermal Analysis

\begin{tabular}{llll}
\hline DNC & $\mathbf{T g}\left(\mathbf{c}^{\mathbf{0}}\right)$ & $\mathbf{T M}\left(\mathbf{c}^{\mathbf{0}}\right)$ & $\mathbf{T C}\left(\mathbf{c}^{\mathbf{0}}\right)$ \\
\hline A1 & 110 & 584 & 150.3 \\
A2 & 111 & 580 & 145.4 \\
A3 & 106 & 584 & 138 \\
\hline
\end{tabular}

\section{DNC Dental Nano Composite}

Tg: Degree glass transition

Tm: Melting Point

Tc: Degree of crystallization 


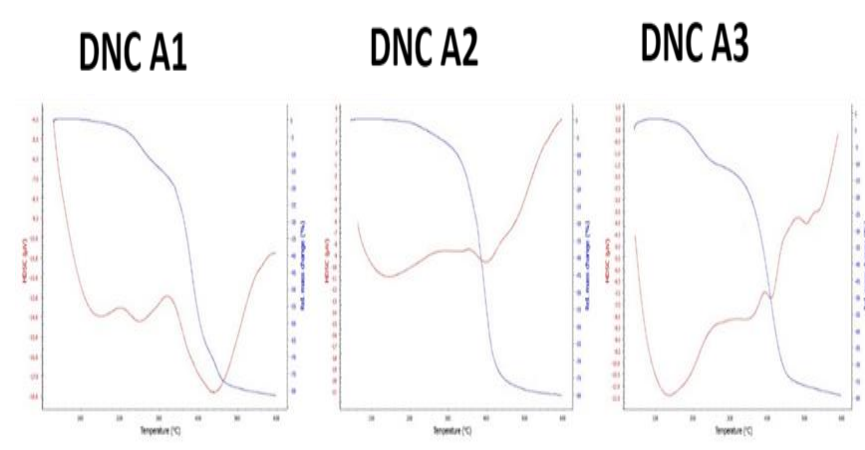

Fig 6: TGA and DSC forA1) composite dental with $\mathrm{ZrO}_{2}$, A2) composite dental with $\mathrm{SiO} 2$ and $\mathrm{A}_{3}$ ) composite dental with $\mathrm{HA}$.

\subsubsection{Flexural Strength and Elastic Modulus}

The main objective in this study is to study the flexural strength of the composite samples containing various mass fractions of nano-fillers $\left(\mathrm{SiO}_{2}\right.$, $\mathrm{ZrO}_{2}$ and hydroxyapatite) as shown in Fig.7. There is an initial increase in the flexural strength of the nano-particles filled composites as the filler content is increased from ( 0.66 to 8$) \mathrm{wt} . \%$. However, a decrease in strength values is observed at higher values of filler addition. Going back to the results obtained for the flexural strength, it should be reminded that, one of the major factors affecting the mechanical characteristics of composite samples is extent of interfacial interaction. In other words, poor interfacial interactions prevent a sufficient transfer of stress between components. In such cases, the addition of filler particles is expected to increase the number of weak links and therefore have a negative impact on strength. Therefore, we believe that the increase in the strength shown in Fig. 7 is due to the reduced macromolecular mobility in the matrix and the drop in the strength. Values are most likely attributable to the overcoming effect of poor filler/matrix coherence at higher values of the filler content.

Spherical and evenly distributed micrometer size filler particles can be observed and a closer examination of this micrograph indicates that, as a result of a strong interfacial bond between the filler and resin, some filler particles have been effectively forced out of the matrix during the mechanical testing. The modulus of elasticity values were calculated from these data and are presented in Fig. 7. As indicated in this table, except for the case of the sample containing $4.6 \mathrm{wt} . \%$ nano fillers the increase in the content of the filler is accompanied with increased value of the elastic modulus. A possible explanation for the increased value of elasticity modulus is the more restricted motion of Bis-GMA matrix with an increase in the filler content [35]. Once again, at some critical value of the filler content the sample behavior is dominated by the poor bonding at the interface causing a decrease in modulus value. An exponential dependence of modulus of elasticity on filler content has already been reported in the literature [36]. Another point which can be concluded from the data is that there is no sign of plastic deformation prior to fracture in these samples.

These results show that all nano-particulate containing samples have significantly higher mean strength than those of the nano-filled composite samples 1. This implies an effective stress transfer as a result of good bonding between the nano-sized particles and the matrix. It is also important to take into account that the filler fraction values were lower in the nano-size filler containing composites compared to the nano-filled composite samples prepared in this study. In summary, the data presented in this study on the mechanical properties of the nano-sized filler containing samples, indicates that in spite of the lower weight fraction, the use of nanosize nano-fillers particles resulted in a considerable enhancement in the mechanical properties of the dental restorative composite samples. Obviously, several factors are at play, but we believe that maybe the most important is the favorable adhesion between the nano-sized filler and the polymeric matrix. Incorporating of nano-fillers in dental composites enhanced the flexural strength by over $100 \%$. The flexural strength enhancement may be a result of increased area of the surface of the particles of the filler due to the reduced size of the particle that produces high energy of the surface at interface of the filler-matrix. This results from the capability of those nano-particles in hindering the propagation of cracks within the matrix of the dental composite based on the mechanism of the strengthening, in addition to strong bonding between those particles and the resin matrix. Adding $\mathrm{HA}, \mathrm{SiO}_{2}$, and zirconium oxide as nano-particles leads in increasing both dental nano-composites' flexural modulus and strength. The cause of this kind of behavior is that high shear strength of the interface, between matrix of the resin and the nano-particles of each of $\mathrm{HA}, \mathrm{SiO}_{2}$, and zirconium oxide associated with the creation of physical cross-link bonding shielding or covering nano-particles, which successively prevent cracks from propagating inside the material, according to foregoing.

In addition to that, incorporating brittle nano-particles in the matrix of the polymer enhances composite stiffness with the restriction of polymer chain mobility in nano-composite. and sufficient nano-particle distribution, particularly at low nano-particle additive percentages to composite materials, as it has been performed in the present study nano-particle content's volumetric fraction in composites not higher than $18 \%$ ratio) which decreases nano-particle agglomeration in the composite, which could result in the reduction from location and the density of stresses are insufficient for breaking weak interface interactions. Which is why, those small concentrations of the stress may be transferred easily from the matrix to brittle nano-particles, thereby permitting particles in contributing their property of the high brittleness to composites therefore, it result in increasing both flexural modulus and strength.

\subsubsection{Compressive Strength}

The strength of nano-fillers reinforcement resin which is under compressive loading has been evaluated with the approach which is used to test nano-fillers. The values were stated in Fig. 8 for compressive strength values of synthetic polymer which is reinforced by nano-fillers of a variety of average size of particles. Some other materials, which are presently in general utilization, have been illustrated for comparisons. Specimens made by nano-fillers had high compressive strengths which gives the confirmation of the existence of differences that are statistically significant between all groups of samples. Moreover, the results of the compressive test have shown the differences amongst samples $\mathrm{SiO}_{2}(10-30 \mathrm{~nm})<$ $\mathrm{ZrO} 2(20-30) \mathrm{nm}<\mathrm{HA}(60) \mathrm{nm}$. The testing of compressive strength is utilized to assess mechanical characteristics of this type of material. Due to the fact that the majority of the masticator loads belong to compressive forces class. There is a considerable importance in evaluating the resin material durability in that conditions [37]. Advancements that have been accomplished in the area of nano-technology had a great influence on resins composition [38]. Many different types of composite were produced and presented according to the nano-technology. There is an importance of compressive strength measuring in vitro research which were characterized as sufficient indicators for the simulation of functional forces which have loaded upon the restorative materials that are under mastication $[39,40]$. Consequently, in the present work, nano-composite resin's compressive strength has been projected. Composite resins that are nano-filled combined with nano-cluster formulations that reduce filler particles' interstitial spacing to nano-sized particles presents higher loading of the filler, advanced physical characteristics, in comparison to these complexes. The 
average sizes of filler particles range between $10 \mathrm{~nm}$ and $30 \mathrm{~nm}$. Spherical shapes include numerous benefits such as improvements in the filler load of the composites as well as increasing fracture strengths due to the fact that mechanical stresses tend to be concentrated on filler particles angles and protrusions. The majority of the nano-composites have filler particles that are spherically-shaped with improved filler load. In the present research, nano-composites have higher compressive strengths compared to compressive strengths of the other dental-composite. The present research agrees in increased strength value of the nano-composite resin. It has appeared that besides greater sizes of filler particles in dental composite resins, the existence of $\mathrm{SiO}_{2}$, hydro-apatite, and zirconium fillers have a significant role in its enhanced strength. The existence of aromatic cycles in the monomers such as Bisphenol di-methacrylate and Bis-GMA has been detected in dental composites results in reducing cyclizing and increasing cross linking in the polymer and provides consecutive enhancement in strength/durability and mechanical characteristics. None-the-less, in acrylate and TEGDMA monomers particularly as a result of its high flexibility, there's more chance of intermolecular cyclizing. In a way that Bis-GMA stiffness is a factor in improving compressive strength as well [41]. Esthetic properties of the nano-composites are analogous to the esthetic characteristics of the natural teeth. Due to the fact that they are of a sufficient wear resistance they result in no enamel wear of analogous teeth. Their polymerization shrinkage is quite minor, which is why, they have less tension which results in decreasing of the over-sensitivity of postop. there are filler particles in the nano-composites, and they enhance the strength of the matrix and produce higher toughness to fractures [42, 43]. Therefore, nano-composites of high compressive strength that utilized in the present research may be clarified with their higher loadings of the filler. Numerous researches reported the correlation between the volume fraction and the mechanical characteristics of the fillers [44-46].

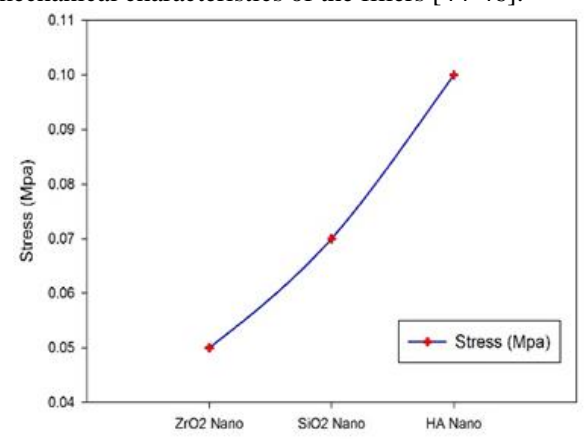

Figure 7: Show the flexural strength of Nanofiller-reinforced composite dental

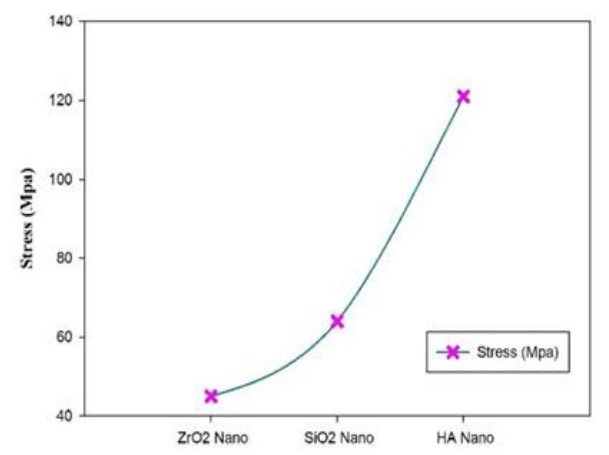

Figure 8: Compressive strength of Nano-filler- reinforced composite dental

\section{Conclusions}

This work has been focused on synthesizing a new Bis-GMA-based composite resin with nano-fillers $\left(\mathrm{HA}, \mathrm{SiO}_{2}\right.$, and $\mathrm{ZrO}_{2}$ ) for the use in the field of dentistry as restorative material. The filler that has been newly synthesized was a powder of white color, with carefully chosen particle sizes. The use of nano-sized fillers particles has proven highly effective in the improvement of mechanical characteristics. Another benefit of the nano-size reinforcement agents in comparison with the micro-sized particles has been the lower loading requirement. The enhanced mechanical characteristics which are shown by the complexes that include nanoparticles is mostly a result of more efficient interaction of filler/polymer. A nano-particle dispersion of higher efficiency, in combination with higher loadings could result in a more enhanced complex dental restorative material. Additional researches are required for improving interactions between nano-sized fillers and matrix phase and in addition to that, increasing nano-filler particles' loading capacity to the matrix of the polymer. In spite of the limitations of the present study, it might be concluded that dental nano-composites that are synthesized have several advantages, such as better water resistance and lower volumetric shrinkage even the dental composite water solubility was small.

\section{REFERENCES}

[1] J. Mutter, "Is dental amalgam safe for humans? The opinion of the scientific committee of the European Commission," Journal of Occupational Medicine and Toxicology, 6(1), 2, 2011 .

[2] A. Lindberg, "Resin composites, sandwich restorations and curing techniques (dissertation)," Umeå, Sverige: Umeå universities, 2005.

[3] N. Ilie, R. Hickel, "Resin composite restorative materials," Aust Dent J;56 Suppl 1:59-66, 2011.

[4] KJ . Anusavice, C. Shen, HR. Rawl, "Phillips' Science of dental materials," 12th rev. ed. St. Louis: Elsevier, 276-96,2013.

[5] European Commission, Definition of a nanomaterial ,2014.

[6] A. Tiba, DG. Charlton, KS. Vandewalle, JC Jr. Ragain, "Comparison of two video-imaging instruments for measuring volumetric shrinkage of dental resin composites," J Dent 33:757-63, 2005.

[7] B. Han, Y. Dong, X. Gao, X. Wang, F. Tian, "Effect of filler content on the micro tensile bond strength of composite resin and dentin in Class I cavities," Quintessence Int ,43:16-22,2012.

[8] CJ. Kleverlaan, AJ. Feilzer, "Polymerization shrinkage and contraction stress of dental resin composites," Dent Mater; 21: 1150-1157, 2005.

[9] R. Labella, P. Lambrechts, B. Van Meerbeek, G. Vanherle , "Polymerization shrinkage and elasticity of flowable composites and filled adhesives," Dent Mater; 15: 128-13, 1999.

[10] MM. Stavridakis, D. Dietschi, I. Krejci, "Polymerization shrinkage of flowable resin-based restorative materials," Operative Dent , 30: 118-128, 2005.

[11] I.M. Barszczewska-Rybarek, "Characterization of urethane-dimethacrylate derivatives as alternative monomers for the restorative composite matrix. Dent. Mater. 30, 1336-1344, 2014.

[12] I. Barszczewska-Rybarek, "Structure-property relationships in dimethacrylate networks based on Bis-GMA, UDMA and TEGDMA," Dent. Mater. 25, 1082-1089, 2009.

[13] I. Sideridou, V. Tserki, G. Papanastasiou, "Effect of chemical structure on degree of conversion in light-cured dimethacrylate-based dental resins," Biomaterials 23, 1819-1829, 2002.

[14] E. S. Vinícius ,Gajewski et al, "Monomers Used in Resin Composites: Degree of Conversion, Mechanical Properties and Water Sorption/Solubility,” Braz Dent J , 23(5): 508-514, 2012. 
[15] MT. Lemon, MS. Jones, JW. Stansbury, "Hydrogen bonding interactions in methacrylate monomers and polymers," J Biomed Mater Res A ,83A:734746, 2007.

[16] I. Sideridou, V. Tserki, G. Papanastasiou, "Study of water sorption, solubility and modulus of elasticity of light-cured dimethacrylatebased dental resins," Biomaterials ,24:655-665, 2003.

[17] CS. Pfeifer, ZR. Shelton, RR. Braga, D. Windmoller, JC. Machado, JW. Stansbury, "Characterization of dimethacrylate polymeric networks: A study of the crosslinked structure formed by monomers used in dental composites," Eur Pol J. 47:162-170,2011.

[18] J.W. Stansbury, J.M. Antonucci, "Dimethacrylate monomers with varied fluorine contents and distributions," Dent. Mater. 15, 166-173, 1999.

[19] M.C. Chang, L.D. Lin, F.H. Chuang, C.P. Chan, T.M. Wang, J.J. Lee, P.Y. Jeng, W.Y. Tseng, H.J. Lin, J.H. Jeng, "Carboxylesterase expression in human dental pulp cells: Role in regulation of Bis-GMA-induced prostanoid production and cytotoxicity," Acta Biomater. 8, 1380-1387, 2012.

[20] N. Yamazaki, S. Kurata, "Synthesis of dimethacryloxy ethyl-1,1,6,6tetrahydro-perfluoro-hexamethylene-1,6- dicarbamate as dental base monomers and the mechanical properties of the copolymers of the monomer and methyl methacrylate," Dent. Mater. J. 30, 103-108, 2011.

[21] M. Giannini et al, "Characterization of Water Sorption, Solubility, and Roughness of Silorane- and Methacrylate-based Composite Resins," Operative Dentistry, 39-3, 264-272, 2014.

[22] D.W. Van Krevelen, "Properties of polymer," 3rd ed.; Elsevier, Amsterdam, 1990.

[23] S.H. Dickens, and B.H. Cho, "Interpretation of bond failure through conversion and residual solvent measurements and Weibull analyses of flexural and microtensile bond strengths of bonding agents," Dent. Mater., $21,354-364,2005$.

[24] R.L. Santos, M. Clarke, F. Braden, K.W.M. Guitian and Davy, "Water absorption characteristics of dental composites incorporating hydroxyapatite filler”, Biomaterials, 23: 1897-1904, 2002.

[25] R. Labella, P. Lambrechts, B. Van Meerbeek, G. Vanherle, "Polymerization shrinkage and elasticity of flowable composites and filled adhesives," Dent Mater 15:128-37, 1999.

[26] JW. Stansbury, "Cyclopolymerizable monomers for use in dental resin composites," J Dent Res ,69:844-8, 1990.

[27] AJ. Feilzer, BS. Dauvillier ,"Effect of TEGDMA/BisGMA ratio on stress development and viscoelastic properties of experimental two-paste composites," J Dent Res , 82:824-8, 2003.

[28] JL. Ferracane, EH. Greener, "The effect of resin formulation on the degree of conversion and mechanical properties of dental restorative resins," J Biomed Mater Res 20: 121-31, 1986.

[29] RR. Braga, TJ. Hilton, JL. Ferracane ,"Contraction stress of flowable composite materials and their efficacy as stressrelieving layers," J Am Dent Assoc, 134:721-8,2003.

[30] FC. Calheiros, FT. Sadek, RR. Braga, PEC. Cardoso," Polymerization contraction stress of low-shrinkage composites and its correlation with microleakage in class V restorations," J. Dent 32:407-12, 2004.

[31] A. Tarasov, " thermal analysis: methods, principles, applicason," lecture on thermal analysis, 26:16.2012.

[32] K. B. Cantre, J. H. Martin, K. S. Ro, " Application of Thermogravimetric Analysis for the Proximate Analysis of Livestock Wastes," Journal of ASTM International 7(3)P:2-13, 2009.

[33] R.v. Adivarekar, S.D. dasarwar, N.S. Khurana, "synthesis of halogen free flame retardant and application polypropylene," indian journal of fibre \&textile research 38:pp:9-13, 2012.

[34] B. Wunderlich, "Thermal Analysis, text for an audio course. ATHAS, Advanced Thermal Analysis," Alaboratory for Research and Instruction, 1981.

[35] E. Asmussen, and A. Peutzfeldt, "Mechanical properties of heat treated restorative resins for use in the inlay/onlay technique," Scan. J. Dent. Res., 564-571,1998.

[36] G. Willems, P. Lambrechts ,M. Braem, J. P. Celis, and G. Vanherle, “A classification of dental composites according to their morphological and mechanical characteristics,” Dent. Mater., 8, 310-318, 1992.
[37] JA. Mohandesi, MA. Rafiee, V. Barzegaran, "Compressive fatigue behavior of dental restorative composites," Dent. Mater J. 26: 827-837, 2007.

[38] D. Ure, J. Harris, "Nanotechnology in dentistry: Reduction to practice," Dentistry Update 30: 10-15, 2003.

[39] KJ. Anusavice, "Phillips Science of Dental Materials," Edition t, editor: Saunders, p 832, 2003.

[40] JM. Powers, RL. Sakaguchi, "Craig's restorative dental materials,": Mosby Inc.2006.

[41] K. Ratanapridakul, K. Leinfelder, J. Thomas, "Effect of finishing on the in vivo wear rate of a posterior composite resin," The Journal of the American Dental Association 118(3):333-335, 1989.

[42] JE. Elliot, LG. Lovell, CN . Bowman, "Primary cyclization in the polymerization of bis-GMA and TEGDMA: a modeling approach to understanding the cure of dental resins," Dent. Mater. 17: 221-229, 2002.

[43] A. Yap, "Comparison of surface finish of new aesthetic restorative materials," Oper. Dent. 29:100-104, 2004.

[44] MH. Chen, SP. Sun, WF. Su, "Low shrinkage light curable Nanocomposite for dental restorative material," Dent. Mater., 222: 138-145, 2006.

[45] M. Braem, W. Finger, VE. Van Doren, P. Lambrechts, G. Vanherle, "Mechanical properties and filler fraction of dental composites," Dental Materials 5(5):346-349,1989.

[46] K. Chung, E. Greener ,"Correlation between degree of conversion, filler concentration and mechanical properties of posterior composite resins," Journal of Oral Rehabilitation 17(7): 487-94,1990.

[47] A N. Khaled, "Physical Properties of Dental Resin Nanocomposites," Master thesis, University of Manchester School of Dentistry, 2012. 